\title{
Abrupt spontaneous suprachoroidal hemorrhage post-23-gauge vitrectomy during peritoneal dialysis
}

This article was published in the following Dove Press journal:

Clinical Ophthalmology

12 June 2013

Number of times this article has been viewed

\section{Moosang Kim \\ Seung-Chan Lee \\ Seung-Jun Lee}

Department of Ophthalmology, School of Medicine, Kangwon National University, Chuncheon, South Korea
Correspondence: Seung-Jun Lee Department of Ophthalmology, School of Medicine, Kangwon National University, Chuncheon, South Korea Email ophkimmoo@gmail.com

\begin{abstract}
Herein, we report a case of abrupt suprachoroidal hemorrhage (SCH) that developed during peritoneal dialysis in a patient with proliferative diabetic retinopathy. A 53-year-old female patient visited our clinic with blurred vision due to vitreous hemorrhage and proliferative diabetic retinopathy. Her medical history included diabetes, hypertension, chronic renal failure, and she had received scheduled peritoneal dialysis. No anticoagulant agents were used. We performed combined phacoemulsification with intraocular lens implantation and vitrectomy without any complications. Two hours later, the retina was stable and the intraocular pressure (IOP) was $11 \mathrm{mmHg}$. Four hours later, while receiving peritoneal dialysis, she abruptly developed ocular pain. Examination of her eye revealed an IOP of $38 \mathrm{mmHg}$ and a SCH in the entire peripheral retina and posterior pole. At 12 hours after surgery (on the same day), the $\mathrm{SCH}$ was found to be further aggravated, and because a "kissing retina" was imminent, silicone oil was injected. An attempted fluid-air exchange failed because there was not enough space to fill with silicone oil due to aggravation of the $\mathrm{SCH}$. Sclerotomies were performed to remove the $\mathrm{SCH}$, and to create space for the silicone oil injection. Two months after surgery, the silicone oil was removed and her visual acuity was found to have improved to 20/40, but the patient died of pontine hemorrhage 1 month later. SCH can occur in vitrectomized eyes due to an increase in abdominal pressure during peritoneal dialysis, because chronic renal failure patients with diabetes and hypertension have structural vulnerabilities and vascular weaknesses due to arterial sclerosis in response to the increased blood pressure.
\end{abstract}

Keywords: chronic renal failure, $\mathrm{SCH}$, silicone oil, kissing retina, sclerotomy

\section{Introduction}

Suprachoroidal hemorrhage $(\mathrm{SCH})$ is an accumulation of blood within the "suprachoroidal space," which is a potential space situated between the choroid and the sclera. $\mathrm{SCH}$ has been reported to occur after all types of intraocular procedures. ${ }^{1} \mathrm{SCH}$ has also been reported following the Valsalva maneuver, hemodialysis, and thrombolysis with heparin and low molecular-weight derivatives for myocardial infarction. ${ }^{2,3}$ However, as far as we are aware, there have been no reports of SCH occurring during peritoneal dialysis. Here, we present a case of an abrupt development of $\mathrm{SCH}$ in a patient undergoing peritoneal dialysis after vitrectomy.

\section{Case report}

A 53-year-old female patient presented with sudden visual loss in her right eye. She had been diagnosed with diabetes and hypertension 20 years earlier. Peritoneal dialysis had been ongoing since her renal failure diagnosis the year before. No anticoagulant 
agents were used. She was $151 \mathrm{~cm}$ tall and weighed $98 \mathrm{~kg}$. Best-corrected visual acuity was counting fingers in the right eye and 20/40 in the left eye. The intraocular pressure (IOP) was $19 \mathrm{mmHg}$ in the right eye and $12 \mathrm{mmHg}$ in the left eye. Axial length was $22.77 \mathrm{~mm}$ in the right eye and $22.34 \mathrm{~mm}$ in the left. Anterior segment examination revealed moderate nuclear sclerotic cataracts in each eye, while fundus examination revealed a dense vitreous hemorrhage in the right eye and multiple abnormal new vessels at the inferotemporal retina in the left.

Two days after presentation, we performed combined phacoemulsification with intraocular lens implantation and vitrectomy in her right eye. Vitrectomy was performed using a 23-gauge vitreous cutter driven by an Associate 2500 vitrectomy unit (DORC International, Zuidland, The Netherlands) and the DORC Two Step 23 Gauge Vitrectomy System. During vitrectomy, we identified a posterior vitreous detachment and observed new abnormally raised vessels at the peripheral retina. Panretinal endolaser photocoagulation was thus performed at the end of the surgery. Two hours after the vitrectomy, the IOP was $11 \mathrm{mmHg}$ and no signs of endophthalmitis in the anterior and posterior chambers were observed. Fundus findings were the same at the end of the surgery. Four hours after vitrectomy, she began peritoneal dialysis with $1.5 \%$ Stay-Safe ${ }^{\circledR}$ (Fresenius Medical Care, Bad Hamburg, Germany). The patient usually received four $2 \mathrm{~L}$ exchanges of peritoneal dialysis fluid daily. She complained of sudden onset severe ocular pain and mild dyspnea approximately 10 minutes after infusion of the dialysis solution into the peritoneal cavity.

Slit-lamp examination revealed a shallow anterior chamber. The IOP was $38 \mathrm{mmHg}$ in the right eye. Fundus examination of the right eye revealed an elevated, dark brown choroidal mass located in the entire retina. (Figure 1). Intravenous mannitol was immediately administered, along with systemic acetazolamide, topical dorzolamide/timolol eye drops (Cosopt ${ }^{\circledR}$, Merck, Whitehouse Station, NJ, USA), and brimonidine tartrate (Alphagan ${ }^{\circledR}$-P, Allergan, Irvine, CA, USA). At 12 hours after the vitrectomy (the same day), the $\mathrm{SCH}$ was found to be further aggravated and threatened the macular architecture. A "kissing retina" was imminent. We thus performed vitreous cavity reformation and silicone oil tamponade then a secondary surgery was performed under general anesthesia.

In the surgery, a $6 \mathrm{~mm}$ long infusion cannula was inserted $3.5 \mathrm{~mm}$ posterior to the limbus in an area with less choroidal detachment, an infusion catheter connection inserted, and two cannulae inserted $3.5 \mathrm{~mm}$ posterior to the limbus in the superiotemporal and superionasal quadrants. When we performed the fluid-air exchange, however, there was not enough space to inject the silicone oil because of the $\mathrm{SCH}$. We made a full-thickness scleral incision to drain the $\mathrm{SCH}$ in the areas of highest choroidal detachment. Fluid-air and air-silicone oil exchange were then conducted and endolaser photocoagulation around the peripheral retina was performed (Figure 2). The choroidal elevation improved, and the patient was left with only minor subretinal hemorrhages inferiorly. The retina was flat.

Two months after the vitrectomy, the silicone oil was removed (Figure 3) and the patient's visual acuity had improved to 20/40. However, 1 month later, she died of a pontine hemorrhage.

\section{Discussion}

"SCH" is defined as hemorrhage in the suprachoroidal space that is extensive enough to forcibly extrude the intraocular contents from the eye or to move the retinal surfaces into apposition. Ophthalmic risk factors include glaucoma, aphakia, elevated IOP, axial myopia, and inflammation. ${ }^{4}$ Advanced age, hypertension, and atherosclerosis are recognized systemic risk factors. ${ }^{4}$ There have also been reports of SCH in patients following the Valsalva maneuver ${ }^{5}$ and in those with ocular hypertension and open-angle glaucoma, and high myopia without closed-angle glaucoma. ${ }^{6}$

Rossi et $\mathrm{al}^{7}$ suggested the use of choroidal hemorrhage drainage through a 23-gauge vitrectomy cannula combined with 23-gauge vitrectomy and silicone oil tamponade. More recently, Rezende et $\mathrm{al}^{8}$ evaluated transconjunctival drainage of serous and hemorrhagic choroidal detachment using 20- or 25 -gauge transconjunctival trocar/cannula systems. They inserted the transconjunctival trocar/cannula systems into the suprachoroidal space $7.0 \mathrm{~mm}$ from the limbus, and reported that vitrectomy may not be necessary when the choroidal detachments are drained in this manner. Nadarajah et $\mathrm{al}^{9}$ suggested that early controlled drainage of massive $\mathrm{SCH}$ with an expanding gas bubble (100\% perfluoropropane) could help in achieving a good visual outcome. In their study, the mean drainage time of the $\mathrm{SCH}$ was 3.5 days after onset. The use of $100 \%$ perfluoropropane has the advantage of maintaining positive pressure while facilitating controlled drainage of the hemorrhage as the clot lyses.

This patient had chronic renal failure (CRF) associated with hypertension and diabetes mellitus. CRF is a progressive loss in renal function over a period of 3 months or longer and can be classified into five stages depending on the level of renal function disruption and kidney damage. For those at 

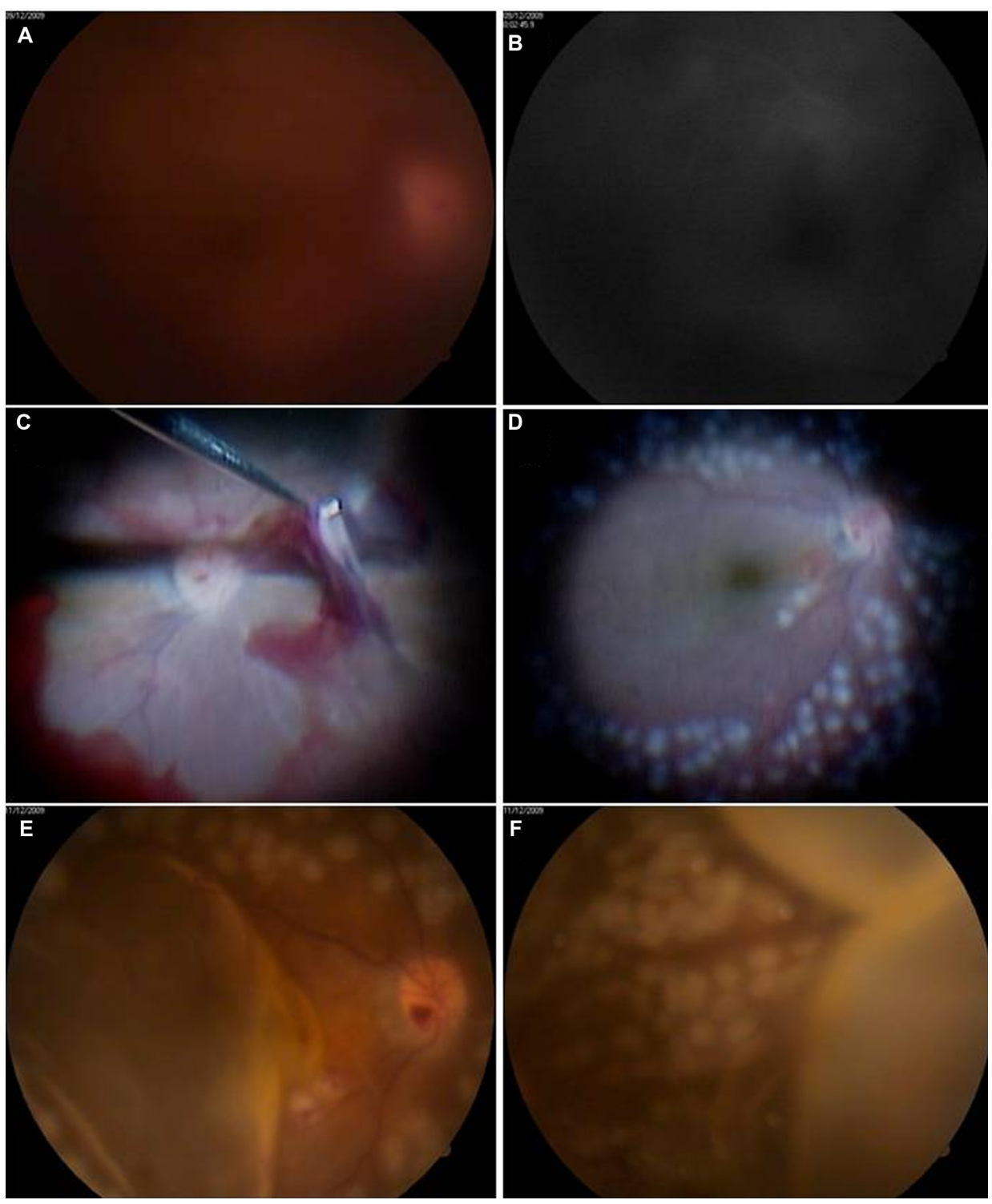

Figure I (A and B) Fundus photography and fluorescein angiography images revealed vitreous hemorrhage in the right eye during preoperative examinations. (C) Intraoperative findings of the first vitrectomy. (D) Fundus photographs showed no complications, including vitreous, at the end of vitrectomy. (E and F) Four hours after surgery, fundus photography showed choroidal detachment at the posterior pole and peripheral retina.

Stage 5, which is end-stage renal disease (ESRD) with less than $15 \%$ of renal function remaining, renal replacement therapies such as hemodialysis, continuous ambulatory peritoneal dialysis, or renal transplantation are required. ESRD requiring dialysis means the renal capillaries are extensively damaged and long-term damage has occurred to the systemic blood vessels. This implies that the blood vessels are vulnerable to changes in blood pressure or shearing forces because ESRD patients tend to have conditions such as blood vessel instability and sclerosis. Choroidal hemorrhage was reported in a patient with CRF in the form of a cough-induced Valsalva maneuver, ${ }^{10}$ and spontaneous progressive $\mathrm{SCH}$ was also reported in a patient undergoing hemodialysis. ${ }^{11}$ These cases clearly demonstrate the vulnerability of the vascular system in patients with ESRD. Anticoagulant and antiplatelet agents used for medical treatment and the vascular system of the patients vulnerable to unstable body fluid shifts and changes in blood pressure during hemodialysis are considered the main cause. $\mathrm{SCH}$ also occurred in a patient receiving ocular surgery under general anesthesia who experienced paroxysmal coughing when the depth of anesthesia was decreased. ${ }^{12}$ Another case was a hemodialysis patient who developed SCH during severe coughing. ${ }^{5}$ In the latter case, the Valsalva maneuver induced by severe coughing was thought to have resulted in $\mathrm{SCH}$. 

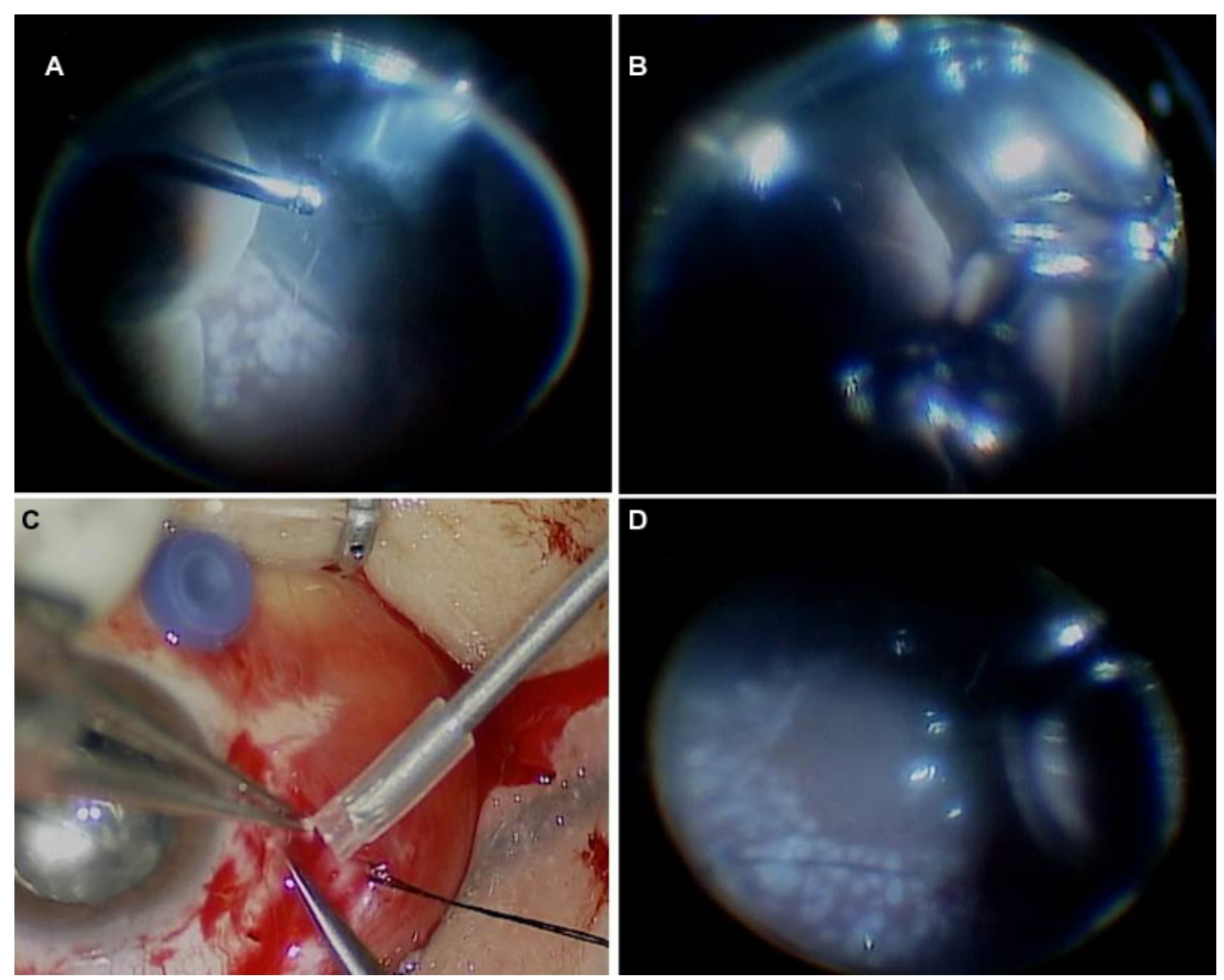

Figure 2 Intraoperative findings of the second vitrectomy. (A) Huge choroidal detachment and (B) "kissing retina" were observed. (C) Fundus photography showed the suprachoroidal hemorrhage removal through the sclerotomy site. (D) At the end of the vitrectomy, fundus photography showed a flat posterior pole and peripheral retina.

In patients with diabetes or hypertension accompanied by arteriosclerosis or vascular injury of the choroidal blood vessels, instability of the choroidal vascularity increases and the blood vessels are vulnerable to a shearing force, which are prime conditions for SCH. Continuous ambulatory peritoneal dialysis is a convenient and economical alternative to hemodialysis because it allows the patient to have dialysis

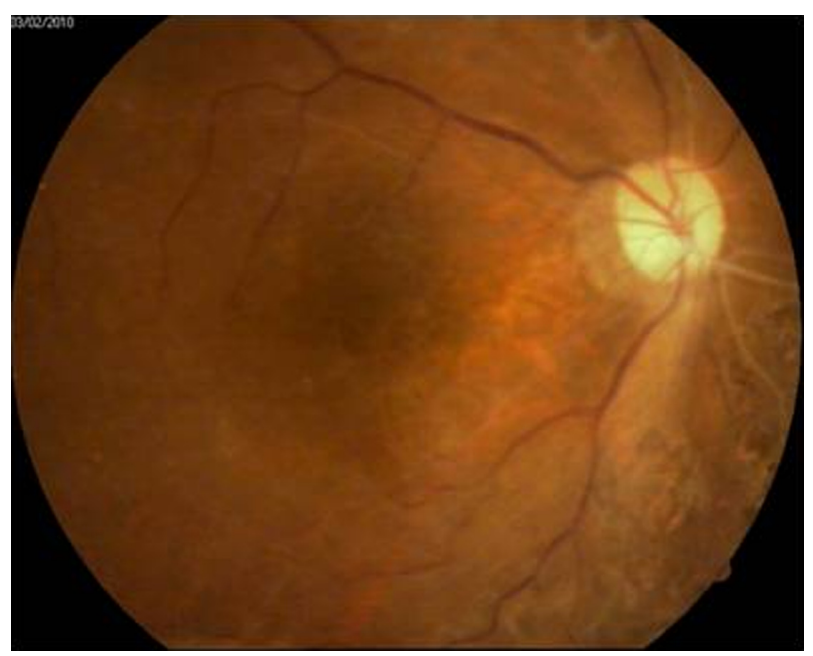

Figure 3 Fundus photography after silicone oil removal. A well-attached retina was observed. without visiting the hospital, thereby minimizing disruption to his or her daily life. Peritoneal dialysis requires a much less restricted diet and water intake than hemodialysis and makes it easier to control blood glucose at the same time. Thus, it is used in approximately $15 \%$ of CRF patients who receive dialysis. ${ }^{13}$ There are also disadvantages, however, such as a greater risk of peritonitis and protein loss compared with hemodialysis, and a risk of elevated intra-abdominal pressure when a large amount of dialysate enters the peritoneal cavity over a short period. ${ }^{14}$ The reason for the increased intra-abdominal pressure during peritoneal dialysis may be the process itself. Approximately $2000 \mathrm{~mL}$ of glucose solution $(1.50 \%-4.25 \%)$ is infused by gravity into the peritoneal cavity over a short period (5-10 minutes) and 4 to 6 hours later it is drained out by gravity over 20 to 30 minutes. This process is repeated three to four times a day. Dialysate used in the process can increase the intra-abdominal pressure, which can in turn induce a hernia and increase the blood pressure. ${ }^{12-14}$ Infusion of the peritoneal dialysate immediately elevates systolic pressure in the carotid artery, induces responsive bradycardia, and then gradually elevates the diastolic pressure of the carotid artery. The mechanism behind the conditions is reported to be venous compression in the intraperitoneal cavity that increases peripheral resistance 
and, in turn, increases the cardiac preload. This may persist for about 1 hour after the peritoneal dialysate is drained out, causing instability in the cardiopulmonary vascular system. ${ }^{15-19}$ The same mechanism was probably responsible for the $\mathrm{SCH}$ in the present case. In other words, the dialysis solution that infused into the peritoneal cavity increased intra-abdominal pressure, inducing an elevation in central blood pressure and responsive bradycardia, which in turn led to instability of weak choroidal vessels, which are vulnerable to shearing forces. The patient had severe abdominal obesity with a presumably high intra-abdominal pressure that was further elevated by the $2000 \mathrm{~mL}$ of infused dialysis solution. Neither anticoagulants nor antithrombotic agents were used in this patient, so the effect of such agents can be excluded from the possible causes.

The visual acuity of the patient improved to 20/40 after removal of the silicone oil and the retina was stabilized, but the patient died 1 month later-just 1 day after the sudden onset of a pontine hemorrhage. Systemic vascular abnormalities may already have been extensively present in the patient's body. Although rare, peritoneal dialysis performed after 23-gauge vitrectomy could be a risk factor for the sudden onset of $\mathrm{SCH}$ in patients with proliferative diabetic retinopathy (PDR).

\section{Conclusion}

$\mathrm{SCH}$ could occur in ESRD patients due to a sudden increase in central blood pressure induced by an elevated intraabdominal pressure during peritoneal dialysis following vitrectomy. As such, it might be safer to reduce the amount of dialysis solution or to slow down the infusion rate of the solution to prolong the time to complete peritoneal dialysis in patients who just underwent surgery, such as vitrectomy.

\section{Disclosure}

The authors report no conflicts of interest in this work.

\section{References}

1. van Meur JC, van den Bosch WA. Suprachoroidal hemorrhage following a valsalva maneuver. Arch Ophthalmol. 1993;111(8):1025-1026.

Clinical Ophthalmology

\section{Publish your work in this journal}

Clinical Ophthalmology is an international, peer-reviewed journal covering all subspecialties within ophthalmology. Key topics include: Optometry; Visual science; Pharmacology and drug therapy in eye diseases; Basic Sciences; Primary and Secondary eye care; Patient Safety and Quality of Care Improvements. This journal is indexed on Submit your manuscript here: http://www.dovepress.com/clinical-ophthalmology-journal
2. Barsam A, Heatley CJ, Herbert L. Spontaneous suprachoroidal hemorrhage secondary to thrombolysis for the treatment of myocardial infarction. Clin Experiment Ophthalmol. 2006;34(2):177-179.

3. Wong JS. Spontaneous suprachoroidal haemorrhage in a patient receiving low-molecular-weight heparin (fraxiparine) therapy. Aust $N Z \mathrm{~J}$ Ophthalmol. 1999;27(6):433-434.

4. Chu TG, Green RL. Suprachoroidal hemorrhage. Surv Ophthalmol. 1999;43(6):471-486.

5. Hammam T, Madhavan C. Spontaneous suprachoroidal haemorrhage following a valsalva manoeuvre. Eye (Lond). 2003;17(2):261-262.

6. Chak M, Williamson TH. Spontaneous suprachoroidal haemorrhage associated with high myopia and aspirin. Eye (Lond). 2003; 17(4):525-527.

7. Rossi T, Boccassini B, Iossa M, Lesnoni G, Tamburrelli C. Choroidal hemorrhage drainage through 23-gauge vitrectomy cannulas. Retina. 2010;30(1):174-176.

8. Rezende FA, Kickinger MC, Li G, Prado RF, Regis LG. Transconjunctival drainage of serous and hemorrhagic choroidal detachment. Retina. 2012;32(2):242-249.

9. Nadarajah S, Kon C, Rassam S. Early controlled drainage of massive suprachoroidal hemorrhage with the aid of an expanding gas bubble and risk factors. Retina. 2012;32(3):543-548.

10. Tajika T, Yokozeki H, Ishimaru K, Naito T, Shiota H. Rare case of choroidal hemorrhage complicated with hypertension due to chronic renal failure. $J$ Med Invest. 2008;55:151-155.

11. Saeed MU, Wong D, Heimann H, Gibran SK. Spontaneous progressive supra-choroidal haemorrhage in a patient undergoing haemodialysis. Graefes Arch Clin Exp Ophthalmol. 2007;245(11):1741-1742.

12. Lim HW, Ko BW, Song YM, Lee BR. Suprachoroidal hemorrhage during pars plana vitrectomy associated with valsalva maneuver. Journal of the Korean Ophthalmological Society. 2008;49(6):1022-1027.

13. Başaran O, Moray G, Yağmurdur MC, Aydoğan C, Karakayali H. Six years of surgical experience with continuous ambulatory peritoneal dialysis at one center. Transplant Proc. 2002;34(6):2039-2040.

14. Jung JW, Ryoo SB, Choe EK, Park KJ. Surgical treatment of hernias in patients undergoing continuous ambulatory peritoneal dialysis. J Korean Surg Soc. 2009;77(5):333-337.

15. Verbeke F, Van Biesen W, Pletinck A, Van Bortel LM, Vanholder R. Acute central hemodynamic effects of a volume exchange in peritoneal dialysis. Perit Dial Int. 2008;28(2):142-148.

16. Gotloib L, Mines M, Garmizo L, Varka I. Hemodynamic effects of increasing intraabdominal pressure in peritoneal dialysis. Perit Dial Int. 1981;1(4):41-43.

17. Gotloib L, Garmizo L, Varka I, Mines M. Reduction of vital capacity due to increased intra-abdominal pressure in peritoneal dialysis. Perit Dial Int. 1981;1(5):63-64.

18. Boon D, Bos WJ, van Montfrans GA, Krediet RT. Acute effects of peritoneal dialysis on hemodynamics. Perit Dial Int. 2001; 21(2):166-171.

19. McIntyre CW. Acute cardiovascular functional effects of peritoneal dialysis: what do we know and why might it matter? Perit Dial Int. 2008;28(2):123-125.
PubMed Central and CAS, and is the official journal of The Society of Clinical Ophthalmology (SCO). The manuscript management system is completely online and includes a very quick and fair peer-review system, which is all easy to use. Visit http://www.dovepress.com/ testimonials.php to read real quotes from published authors. 